\title{
Complete pairs of coanalytic sets
}

\author{
by
}

\author{
Jean Saint Raymond (Paris)
}

\begin{abstract}
Let $X$ be a Polish space, and let $C_{0}$ and $C_{1}$ be disjoint coanalytic subsets of $X$. The pair $\left(C_{0}, C_{1}\right)$ is said to be complete if for every pair $\left(D_{0}, D_{1}\right)$ of disjoint coanalytic subsets of $\omega^{\omega}$ there exists a continuous function $f: \omega^{\omega} \rightarrow X$ such that $f^{-1}\left(C_{0}\right)=D_{0}$ and $f^{-1}\left(C_{1}\right)=D_{1}$. We give several explicit examples of complete pairs of coanalytic sets.
\end{abstract}

1. Introduction and notations. All spaces we consider in this note are metrizable and separable, and often zero-dimensional, hence embeddable in the Cantor set $2^{\omega}$ or in the Baire space $\omega^{\omega}$. For unexplained notations we refer to [5].

We will denote by $\mathbb{Q}$ the dense countable subset of $2^{\omega}$ defined by

$$
\mathbb{Q}:=\left\{\alpha \in 2^{\omega}: \exists n \forall p \geq n \quad \alpha(p)=0\right\} .
$$

For any space $X$ we denote by $\mathscr{K}(X)$ the hyperspace of non-empty compact subsets of $X$ equipped with the Vietoris topology. It is a well-known fact that $\mathscr{K}(\mathbb{Q})$ is complete coanalytic. We will also denote by $\mathscr{K}_{\omega}(X)$ the subspace of $\mathscr{K}(X)$ consisting of the countable compact subsets of $X$, and by $\mathscr{K}^{*}(\mathbb{Q})$ the subset

$$
\mathscr{K}^{*}(\mathbb{Q})=\left\{K \in \mathscr{K}\left(2^{\omega}\right): K \backslash \mathbb{Q} \text { has a unique element }\right\}
$$

of $\mathscr{K}\left(2^{\omega}\right)$, which is clearly disjoint from $\mathscr{K}(\mathbb{Q})$ and contained in $\mathscr{K}_{\omega}\left(2^{\omega}\right)$.

We will denote by Seq the countable set $\omega^{<\omega}$ of finite sequences of integers and by $\mathscr{T}$ the set of trees on $\omega$, which we identify with a closed subset

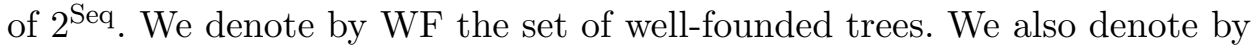
WF $^{*}$ the subset of $\mathscr{T}$ consisting of the trees which have a unique branch. For any tree $T \in \mathscr{T}$ we denote by $\lceil T\rceil$ the set of branches of $T$ and identify it with a closed subset of $\omega^{\omega}$. We will always identify a finite sequence of elements of $\omega \times \omega$ with a pair $(s, t)$ of finite sequences of integers having the

2000 Mathematics Subject Classification: Primary 54H05; Secondary 03E15, 28A05.

Key words and phrases: coanalytic sets, bianalytic functions, functions with closed graph. 
same length. So any tree $T$ on $\omega \times \omega$ is viewed as a subset of Seq $\times$ Seq and for every $x \in \omega^{\omega}$ the set

$$
T(x):=\{s \in \text { Seq }: \exists t \prec x|s|=|t| \text { and }(t, s) \in T\}
$$

is a tree on $\omega$. Moreover it is clear from the definition that the mapping $x \mapsto T(x)$ is then continuous from $\omega^{\omega}$ to $\mathscr{T}$.

Recall that a function $f: X \rightarrow Y$ is said to be bianalytic (we write $\left.f \in \boldsymbol{\Delta}_{1}^{1}\right)$ if $f^{-1}(V)$ is a (relative) coanalytic subset of $X$ for every open subset $V$ of $Y$ (or equivalently for every (relative) coanalytic subset $V$ of $Y$ ). Of course all Borel functions are $\boldsymbol{\Delta}_{1}^{1}$. Moreover the composition of two $\boldsymbol{\Delta}_{1}^{1}$ functions is $\Delta_{1}^{1}$ too.

Recall that a continuous function $f: X \rightarrow Y$ reduces a subset $D$ of $X$ to a subset $C$ of $Y$ if $D=f^{-1}(C)$, and that a coanalytic subset $C$ of the Polish space $Y$ is said to be $\Pi_{1}^{1}$-complete (or simply complete for short if there is no ambiguity) if every coanalytic subset $D$ of a zero-dimensional Polish space $X$ is continuously reducible to $C$ (i.e. if there is a continuous function $f: X \rightarrow Y$ which reduces $D$ to $C$ ). There are many "natural" examples of complete coanalytic sets, namely WF in $\mathscr{T}, \mathscr{K}(\mathbb{Q})$ in $\mathscr{K}\left(2^{\omega}\right)$ or $\mathscr{K}_{\omega}\left(2^{\omega}\right)$ in $\mathscr{K}\left(2^{\omega}\right)$. It is a well-known fact that, under the axiom of analytic determinacy, any coanalytic non-Borel subset of $\omega^{\omega}$ or of $2^{\omega}$ is $\boldsymbol{\Pi}_{1}^{1}$-complete. Moreover it is a classical result of Harrington ([3]) that the converse is true: if any non-Borel coanalytic subset of $2^{\omega}$ is $\Pi_{1}^{1}$-complete, then the analytic determinacy holds.

The main goal of this note is to give similar results for disjoint pairs of coanalytic subsets in a Polish space. Let $X$ and $Y$ be two Polish spaces, $\left(C_{0}, C_{1}\right)$ be a pair of disjoint coanalytic subsets of $Y$ and $\left(D_{0}, D_{1}\right)$ be a pair of disjoint coanalytic subsets of $X$. We will say that the pair $\left(D_{0}, D_{1}\right)$ is continuously reducible to $\left(C_{0}, C_{1}\right)$ if there exists a continuous function $f: X \rightarrow Y$ such that $f^{-1}\left(C_{0}\right)=D_{0}$ and $f^{-1}\left(C_{1}\right)=D_{1}$. Just as for complete coanalytic sets we define complete pairs of coanalytic sets.

Definition 1 . We say that the pair $\left(C_{0}, C_{1}\right)$ is complete if every disjoint pair $\left(D_{0}, D_{1}\right)$ of coanalytic subsets of a zero-dimensional Polish space $X$ (or equivalently of a closed subspace of $\left.\omega^{\omega}\right)$ is continuously reducible to $\left(C_{0}, C_{1}\right)$.

We will study the links between Borel separability and completeness for pairs of coanalytic subsets. We will also give several "natural" examples of complete pairs of coanalytic subsets, and this will lead us to find a characterization of bianalytic functions. Finally, we will give extensions of the previous results to disjoint sequences of coanalytic sets.

2. Some coanalytic sets. The following two theorems have been known for a long time (see for example [2] and the references therein). 
Theorem 2. Let $X$ and $Y$ be Polish, $Z$ be a subspace of $X$ and $f$ : $Z \rightarrow Y$ be bianalytic. Then there exists a coanalytic subset $C$ of $X$ containing $Z$ and a bianalytic function $\widetilde{f}: C \rightarrow Y$ extending $f$.

Let $Y$ and $Z$ be Polish spaces and $X$ be a subspace of $Y \times Z$. We will denote by $U(X)$ the set of elements $y$ of $Y$ such that the fiber $X(y):=$ $\{z \in Z:(y, z) \in X\}$ is a singleton.

Theorem 3. Let $Y$ and $Z$ be Polish spaces and $X$ be a Borel subset of $Y \times Z$. Then the set $U(X)$ is coanalytic and the function $g: U(X) \rightarrow Z$ defined by $\{g(y)\}=X(y)$ is bianalytic on $U(X)$.

Proof. We give the proof for completeness. Denote by $\pi: Y \times Z \rightarrow Y$ the first projection: $(y, z) \mapsto y$. We prove first that the function $g$ is bianalytic on $U(X)$. Indeed, let $V$ be an open subset of $Z$. We have

$$
g^{-1}(V)=U(X) \backslash \pi\left(X \cap\left(Y \times V^{\mathrm{c}}\right)\right),
$$

which is coanalytic in $U(X)$ since $\pi\left(X \cap\left(Y \times V^{\mathrm{c}}\right)\right) \in \boldsymbol{\Sigma}_{1}^{1}$. Thus $g$ is bianalytic.

Using Theorem 2 we get a coanalytic subset $C$ of $Y$ containing $U(X)$ and a bianalytic extension $\widetilde{g}: C \rightarrow Z$ of $g$. The set $X_{2}$ of points of $Y$ where the fiber has at least two points is

$$
\left\{y \in Y: \exists z, z^{\prime} \quad z^{\prime} \neq z \text { and }(y, z) \in X \text { and }\left(y, z^{\prime}\right) \in X\right\},
$$

hence is analytic. Furthermore,

$$
U(X) \subset\left\{y \in C \backslash X_{2}: \widetilde{g}(y) \in X\right\} \subset U(X),
$$

and this shows that $U(X) \in \Pi_{1}^{1}$ since the inverse image of the Borel set $X$ under $\widetilde{g}$ is coanalytic in the coanalytic set $C \backslash X_{2}$.

Again for completeness we now give the proofs of the classical facts that $\mathscr{K}^{*}(\mathbb{Q}), \mathscr{K}_{\omega}\left(2^{\omega}\right) \backslash \mathscr{K}(\mathbb{Q})$ and $\mathrm{WF}^{*}$ are all coanalytic.

Theorem 4. The set $\mathscr{K}^{*}(\mathbb{Q})$ is $\boldsymbol{\Pi}_{1}^{1}$ in $\mathscr{K}\left(2^{\omega}\right)$.

Proof. Let $X=\left\{(K, \alpha) \in \mathscr{K}\left(2^{\omega}\right) \times 2^{\omega}: \alpha \in K\right.$ and $\left.\alpha \notin \mathbb{Q}\right\}$. Then $X$ is clearly a $\Pi_{2}^{0}$ subset of the compact space $\mathscr{K}\left(2^{\omega}\right) \times 2^{\omega}$, and since $\mathscr{K}^{*}(\mathbb{Q})=$ $U(X)$ the conclusion follows from Theorem 3 .

Theorem 5. The set $\mathrm{WF}^{*}$ is $\boldsymbol{\Pi}_{1}^{1}$ in $\mathscr{T}$.

Proof. Let $X=\left\{(T, \alpha) \in \mathscr{T} \times \omega^{\omega}: \alpha \in\lceil T\rceil\right\}$. Then $X$ is clearly a closed subset of the Polish space $\mathscr{T} \times \omega^{\omega}$, and since $\mathrm{WF}^{*}=U(X)$ the conclusion follows again from Theorem 3 .

Theorem 6. The set $\mathscr{K}_{\omega}\left(2^{\omega}\right) \backslash \mathscr{K}(\mathbb{Q})$ is $\Pi_{1}^{1}$ in $\mathscr{K}\left(2^{\omega}\right)$.

Proof. The proof of the following lemma can be found in [4] (Exercise 39.23 , p. 368). 
Lemma 7. There is a countable family $\left(C_{u}\right)$ of coanalytic subsets of $\mathscr{K}_{\omega}\left(2^{\omega}\right)$ and a family $\left(f_{u}\right)$ of $\boldsymbol{\Delta}_{1}^{1}$ functions from $C_{u}$ to $2^{\omega}$ such that for every countable compact subset $K$ of $2^{\omega}$,

$$
K=\left\{f_{u}(K): K \in C_{u}\right\} .
$$

Proof. Consider the following open game $G^{*}(K)$ with parameter $K \in$ $\mathscr{K}\left(2^{\omega}\right)$ : Player II begins by playing some $s_{0} \in 2^{<\omega}$, Player I answers $a_{0} \in$ $\{0,1\}$, Player II plays $s_{1} \in 2^{<\omega}$ and Player I answers $a_{1} \in\{0,1\}$, and so on. And Player II wins the run $\left(s_{0}, a_{0}, s_{1}, a_{1}, \ldots\right)$ iff the infinite dyadic sequence $s_{0} \frown a_{0} \frown s_{1} \frown \ldots \frown s_{n} \frown a_{n} \frown \ldots$ belongs to $K$.

If Player II has a winning strategy $\tau$ in $G^{*}(K)$, this strategy defines a continuous mapping $f: 2^{\omega} \rightarrow K$ by

$$
f(\alpha)=s_{0} \frown \alpha(0) \frown s_{1} \frown \alpha(1) \frown \ldots
$$

where $\left(s_{0}, s_{1}, \ldots\right)$ is the answer by $\tau$ to $\alpha$. It is easily checked that $f$ is one-to-one, hence that $K$ is uncountable if Player II has a winning strategy.

Conversely, if Player I has a winning strategy $\sigma$ in $G^{*}(K)$ we consider the set $S_{\sigma}$ of finite sequences $u=\left\langle s_{0}, a_{0}, s_{1}, a_{1}, \ldots, s_{n-1}, a_{n-1}\right\rangle$ such that for all $p<n, a_{p}$ is the answer by $\sigma$ to $\left(s_{0}, s_{1}, \ldots, s_{p}\right)$, and define $\chi(u):=$ $s_{0} \frown a_{0} \frown s_{1} \frown a_{1} \frown \ldots \frown a_{n-1}$ whenever $u=\left\langle s_{0}, a_{0}, s_{1}, a_{1}, \ldots, s_{n-1}, a_{n-1}\right\rangle$.

Then, for $u \in S_{\sigma}$, we consider the set

$$
E_{u}:=\left\{\alpha \in 2^{\omega}: \chi(u) \prec \alpha \text { and } \forall v \in S_{\sigma} \quad u \prec v \Rightarrow \chi(v) \nprec \alpha\right\} .
$$

It is easily checked that $E_{u}$ contains at most one point: indeed, if $\chi(u)=\alpha_{\mid p}$ and $u=\left\langle s_{0}, a_{0}, s_{1}, a_{1}, \ldots, s_{n-1}, a_{n-1}\right\rangle$ then for all $s=\langle\alpha(p), \alpha(p+1)$, $\ldots, \alpha(q-1)\rangle$ and $a=\sigma\left(s_{0}, s_{1}, \ldots, s_{n-1}, s\right)$ we have $v:=u \frown\langle s, a\rangle \in S_{\sigma}$, hence $\chi(v) \nprec \alpha$. Thus $\alpha(q)=1-a$. This allows us to find inductively the coordinates of $\alpha$ from $\sigma$.

Moreover, if $\alpha \notin \bigcup_{u \in S_{\sigma}} E_{u}$, one can construct inductively a sequence $\left(u_{n}\right)$ of elements of $S_{\sigma}$ such that $u_{0}=\emptyset, u_{n} \prec u_{n+1}$ and $\chi\left(u_{n}\right) \prec \alpha$ for all $n$ : indeed, if $u_{n}$ is defined and satisfies $\chi\left(u_{n}\right) \prec \alpha$ then since $\alpha \notin E_{u_{n}}$ there exists $u_{n+1} \in S_{\sigma}$ such that $u_{n} \prec u_{n+1}$ and $\chi\left(u_{n+1}\right) \prec \alpha$. And since Player I wins the corresponding infinite run in $G^{*}(K)$ we conclude that $\alpha \notin K$. So

$$
K \subset \bigcup_{u \in S_{\sigma}} E_{u}
$$

and $K$ is countable. It follows that $G^{*}(K)$ is won by Player I if and only if $K$ is countable.

The set $\left\{\left(K, s_{0}, a_{0}, s_{1}, a_{1}, \ldots\right)\right.$ : Player I wins the game $\left.G^{*}(K)\right\}$ is open in the Polish space $\mathscr{K}\left(2^{\omega}\right) \times \mathrm{Seq}^{\omega} \times 2^{\omega}$. It follows by Martin's theorem that the set $\mathscr{K}_{\omega}\left(2^{\omega}\right)$ of those $K$ such that Player I has a winning strategy in $G^{*}(K)$ is $\Pi_{1}^{1}$ and that there exists a bianalytic function $K \mapsto \sigma_{K}$ defined 
on $\mathscr{K}_{\omega}\left(2^{\omega}\right)$ assigning to each countable compact subset $K$ of $2^{\omega}$ a winning strategy in the game $G^{*}(K)$.

Then for each sequence $u=\left\langle s_{0}, a_{0}, s_{1}, a_{1}, \ldots, s_{n-1}, a_{n-1}\right\rangle$ the set

$$
C_{u}^{\prime}:=\left\{K \in \mathscr{K}_{\omega}\left(2^{\omega}\right): u \in S_{\sigma_{K}}\right\}
$$

is coanalytic. The function $f_{u}$ defined on $C_{u}^{\prime}$ by $f_{u}(K)=\beta \in 2^{\omega}$ where $\chi(u) \prec \beta$ and, for $q \geq p:=|\chi(u)|$,

$$
\beta(q)=1-\sigma_{K}\left(s_{0}, s_{1}, \ldots, s_{n-1},\langle\beta(p), \beta(p+1), \ldots, \beta(q-1)\rangle\right)
$$

depends continuously on $\sigma_{K}$, hence is bianalytic on $C_{u}^{\prime}$. It follows that $C_{u}:=$ $\left\{K \in C_{u}^{\prime}: f_{u}(K) \in K\right\}$ is coanalytic and that every countable $K$ in $\mathscr{K}\left(2^{\omega}\right)$ satisfies

$$
K=\left\{f_{u}(K): K \in C_{u}\right\}
$$

this completes the proof of the lemma.

It follows from the previous lemma that $K \in \mathscr{K}_{\omega}\left(2^{\omega}\right) \backslash \mathscr{K}(\mathbb{Q})$ if and only if there is some $u=\left\langle s_{0}, a_{0}, s_{1}, a_{1}, \ldots, s_{n-1}, a_{n-1}\right\rangle$ such that $K \in C_{u}$ and $f_{u}(K) \notin \mathbb{Q}$, hence

$$
\mathscr{K}_{\omega}\left(2^{\omega}\right) \backslash \mathscr{K}(\mathbb{Q})=\bigcup_{u} C_{u} \cap f_{u}^{-1}\left(2^{\omega} \backslash \mathbb{Q}\right),
$$

which is a countable union of coanalytic sets, hence coanalytic itself.

3. Borel separation of coanalytic sets. We now compare, for a disjoint pair of coanalytic sets $\left(C_{0}, C_{1}\right)$, the property " $\left(C_{0}, C_{1}\right)$ is complete" with the property " $C_{0}$ is not separable from $C_{1}$ by a Borel set".

LEMMA 8. Let $\left(C_{0}, C_{1}\right)$ be a disjoint pair of coanalytic subsets of $\omega^{\omega}$. If the pair $\left(C_{0}, C_{1}\right)$ is complete then no Borel subset $B$ of $\omega^{\omega}$ can separate $C_{0}$ from $C_{1}$.

Proof. Assume that some Borel set $B$ separates $C_{0}$ from $C_{1}$. Then $B$ is $\boldsymbol{\Sigma}_{\xi}^{0}$ for some countable ordinal $\xi$. If the pair $\left(C_{0}, C_{1}\right)$ were complete then for any Borel subset $B_{1}$ of $\omega^{\omega}$, the pair $\left(B_{1}, \omega^{\omega} \backslash B_{1}\right)$ would be a disjoint pair of coanalytic sets, and there would be a continuous $f: \omega^{\omega} \rightarrow \omega^{\omega}$ such that $f^{-1}\left(C_{0}\right)=B_{1}$ and $f^{-1}\left(C_{1}\right)=\omega^{\omega} \backslash B_{1}$, hence $B_{1}=f^{-1}(B)$; this would imply that $B_{1} \in \Sigma_{\xi}^{0}$, in contradiction with the existence of Borel sets in $\omega^{\omega}$ of arbitrarily large rank.

TheOREM 9. Assume $\operatorname{Det}\left(\boldsymbol{\Sigma}_{1}^{1}\right)$. Let $\left(C_{0}, C_{1}\right)$ be a disjoint pair of coanalytic subsets of $\omega^{\omega}$. Then the pair $\left(C_{0}, C_{1}\right)$ is complete if no Borel subset $B$ of $\omega^{\omega}$ separates $C_{0}$ from $C_{1}$.

Conversely, if any pair of disjoint and Borel non-separable coanalytic sets is complete then the analytic games are determined. 
Proof. Assume that no Borel set separates $C_{0}$ from $C_{1}$ and that $\left(D_{0}, D_{1}\right)$ is a disjoint pair of coanalytic subsets of $\omega^{\omega}$. Consider the game $G$ where Player I and Player II play alternately integers and construct respectively $\alpha \in \omega^{\omega}$ and $\beta \in \omega^{\omega}$ and where Player II wins iff

$$
\begin{array}{rll}
\left(\alpha \in D_{0} \text { and } \beta \in C_{0}\right) & \text { or } \quad\left(\alpha \in D_{1} \text { and } \beta \in C_{1}\right) \\
& \text { or } \quad\left(\alpha \notin D_{0} \cup D_{1} \text { and } \beta \notin C_{0} \cup C_{1}\right) .
\end{array}
$$

Clearly the payoff of this game is the difference of two analytic sets, hence the game $G$ is determined under the assumption of analytic determinacy.

If Player I has a winning strategy $\sigma$, this strategy induces a continuous function $g: \omega^{\omega} \rightarrow \omega^{\omega}$ such that

$g^{-1}\left(D_{0}\right) \cap C_{0}=g^{-1}\left(D_{1}\right) \cap C_{1}=g^{-1}\left(\omega^{\omega} \backslash\left(D_{0} \cup D_{1}\right)\right) \cap\left(\omega^{\omega} \backslash\left(C_{0} \cup C_{1}\right)\right)=\emptyset$.

Then denoting by $C_{0}^{\prime}$ and $C_{1}^{\prime}$ the coanalytic sets $g^{-1}\left(D_{0}\right)$ and $g^{-1}\left(D_{1}\right)$, and by $A$ and $A^{\prime}$ the analytic sets $\omega^{\omega} \backslash\left(C_{0} \cup C_{1}\right)$ and $\omega^{\omega} \backslash\left(C_{0}^{\prime} \cup C_{1}^{\prime}\right)$, we have $A \cap A^{\prime}=\emptyset$. Thus there exists a Borel set $B^{*}$ separating $A$ from $A^{\prime}$. Since $B^{*} \subset C_{0}^{\prime} \cup C_{1}^{\prime}$ and $C_{0}^{\prime} \cap C_{1}^{\prime}=\emptyset$, both $B^{*} \cap C_{0}^{\prime}$ and $B^{*} \cap C_{1}^{\prime}$ are Borel. Moreover, $\omega^{\omega} \backslash B^{*} \subset C_{0} \cup C_{1}$ and $C_{0} \cap C_{1}=\emptyset$, hence $C_{0} \backslash B^{*}$ and $C_{1} \backslash B^{*}$ are both Borel. And it is easily checked that the Borel set

$$
B=\left(B^{*} \cap C_{1}^{\prime}\right) \cup\left(C_{0} \backslash B^{*}\right)
$$

contains $C_{0}$ and is disjoint from $C_{1}$. Thus Player I cannot have a winning strategy if $C_{0}$ and $C_{1}$ cannot be separated by a Borel set.

So Player II has a winning strategy $\tau$ which induces a continuous function $f$ from $\omega^{\omega}$ to $\omega^{\omega}$ such that $f^{-1}\left(C_{i}\right)=D_{i}$ for $i=0,1$, and this shows that every pair $\left(D_{0}, D_{1}\right)$ is reducible to the pair $\left(C_{0}, C_{1}\right)$, hence the pair $\left(C_{0}, C_{1}\right)$ is complete.

We now prove that, as in Harrington's result, the analytic determinacy holds if any pair of coanalytic subsets of $2^{\omega}$ is complete provided it is not Borel separable.

Let $C$ be any non-Borel coanalytic subset of $2^{\omega}, \Gamma$ be a complete coanalytic subset of $2^{\omega}$ and $\varphi$ be a $\Pi_{1}^{1}$-norm on $\Gamma$. We claim that if any non-Borel separable pair of coanalytic subsets of $2^{\omega}$ is complete then $\Gamma$ is continuously reducible to $C$, and by Harrington's theorem this will imply that the analytic determinacy holds. Since $\Gamma$ is $\boldsymbol{\Pi}_{1}^{1}$-complete there exists a continuous function $g: 2^{\omega} \rightarrow 2^{\omega}$ such that $g^{-1}(\Gamma)=C$. Then it is easily checked that the function $\varphi \circ g$ is a $\Pi_{1}^{1}$-norm on $C$ and that the sets

$$
\begin{aligned}
& C_{0}=\left\{(x, y) \in C \times 2^{\omega}: \varphi(g(x)) \leq^{*} \varphi(y)\right\}, \\
& C_{1}=\left\{(x, y) \in 2^{\omega} \times \Gamma: \varphi(y)<^{*} \varphi(g(x))\right\}
\end{aligned}
$$

are disjoint and both coanalytic. Then our claim will follow from the next two lemmas. 


\section{Lemma 10. No Borel set can separate $C_{0}$ from $C_{1}$.}

Proof. Assume towards a contradiction that $B$ is a $\Sigma_{\xi}^{0}$ subset of $2^{\omega} \times 2^{\omega}$ separating $C_{0}$ from $C_{1}$. Let $P$ be a Borel non- $\Pi_{\xi}^{0}$ subset of $2^{\omega}$. Since $\Gamma$ is $\boldsymbol{\Pi}_{1}^{1}$-complete, $P$ is reducible to $\Gamma$, and there is some continuous $f: 2^{\omega} \rightarrow 2^{\omega}$ such that $P=f^{-1}(\Gamma)$. Then $A=f(P)$ is a $\boldsymbol{\Sigma}_{1}^{1}$ subset of $\Gamma$ and the norm $\varphi$ is bounded on $A$ by some ordinal $\eta$. Furthermore, since $C$ is coanalytic non-Borel, the norm $\varphi \circ g$ is unbounded on $C$. Thus there exists some $x \in C$ such that $\eta^{\prime}:=\varphi \circ g(x)>\eta$. Denote by $B^{\prime}$ the $\Pi_{\xi}^{0}$ set $\{y:(x, y) \notin B\}$. Then for $y \in 2^{\omega}$ we have:

$$
\begin{aligned}
& y \in A \Rightarrow \eta^{\prime}>\varphi(y) \Rightarrow(x, y) \in C_{1} \Rightarrow(x, y) \notin B \Rightarrow y \in B^{\prime}, \\
& y \notin \Gamma \Rightarrow \eta^{\prime} \leq^{*} \varphi(y) \Rightarrow(x, y) \in C_{0} \Rightarrow(x, y) \in B \Rightarrow y \notin B^{\prime},
\end{aligned}
$$

hence $A \subset B^{\prime} \subset \Gamma$. Thus $P \subset f^{-1}\left(B^{\prime}\right) \subset f^{-1}(\Gamma)=P$. And we conclude that the $\Pi_{\xi}^{0}$ set $f^{-1}\left(B^{\prime}\right)$ is equal to $P$, a contradiction.

Lemma 11. If the pair $\left(C_{0}, C_{1}\right)$ is complete, then $\Gamma$ is continuously reducible to $C$.

Proof. If $\left(C_{0}, C_{1}\right)$ is complete then the pair $(\Gamma, \emptyset)$ is reducible to $\left(C_{0}, C_{1}\right)$, and there are two continuous functions $f_{0}$ and $f_{1}$ from $2^{\omega}$ to $2^{\omega}$ such that $f=f_{0} \times f_{1}$ reduces $(\Gamma, \emptyset)$ to $\left(C_{0}, C_{1}\right)$. So

$$
\begin{aligned}
& x \in \Gamma \Rightarrow\left(f_{0}(x), f_{1}(x)\right) \in C_{0} \Rightarrow f_{0}(x) \in C, \\
& x \notin \Gamma \Rightarrow\left(f_{0}(x), f_{1}(x)\right) \notin C_{0} \cup C_{1} \Rightarrow f_{0}(x) \notin C,
\end{aligned}
$$

and this shows that $f_{0}$ reduces $\Gamma$ to $C$.

Thus the proof of Theorem 9 is complete.

The following result is well-known.

LEMMA 12. There exist complete disjoint pairs of coanalytic subsets of $2^{\omega}$.

Proof. Let $C$ be a complete $\Pi_{1}^{1}$ subset of $2^{\omega}$. Then $C \times 2^{\omega}$ and $2^{\omega} \times C$ are coanalytic subsets of $2^{\omega} \times 2^{\omega} \simeq 2^{\omega}$, and since the class $\Pi_{1}^{1}$ has the reduction property there are disjoint coanalytic subsets $C_{0}$ and $C_{1}$ of $2^{\omega} \times 2^{\omega}$ such that $C_{0} \subset C \times 2^{\omega}, C_{1} \subset 2^{\omega} \times C$ and $C_{0} \cup C_{1}=\left(C \times 2^{\omega}\right) \cup\left(2^{\omega} \times C\right)$.

We claim that the pair $\left(C_{0}, C_{1}\right)$ is complete. Let $X$ be a zero-dimensional Polish space, and $\left(D_{0}, D_{1}\right)$ a disjoint pair of coanalytic subsets of $X$. Since $C$ is complete there are continuous functions $f, g: X \rightarrow 2^{\omega}$ such that $D_{0}=$ $f^{-1}(C)$ and $D_{1}=g^{-1}(C)$. Then the function $h=f \times g: X \rightarrow 2^{\omega} \times 2^{\omega}$ is continuous and satisfies $h\left(D_{0}\right) \subset C \times\left(2^{\omega} \backslash C\right) \subset C_{0}, h\left(D_{1}\right) \subset\left(2^{\omega} \backslash C\right) \times C \subset C_{1}$ and

$$
h\left(X \backslash\left(D_{0} \cup D_{1}\right)\right) \subset\left(2^{\omega} \backslash C\right) \times\left(2^{\omega} \backslash C\right)=\left(2^{\omega} \times 2^{\omega}\right) \backslash\left(C_{0} \cup C_{1}\right),
$$

which shows that the pair $\left(C_{0}, C_{1}\right)$ is complete. 
4. Bianalytic functions. Our goal is now to give "natural" examples of complete pairs of coanalytic sets. For this we need several results on bianalytic functions.

Lemma 13. Let $X$ be a zero-dimensional Polish space and $\left(C_{0}, C_{1}\right)$ a disjoint pair of coanalytic subsets of $X$. Then there exists a bianalytic function $h: C_{0} \rightarrow \omega^{\omega}$ such that no point of $C_{1} \times \omega^{\omega}$ lies in the closure of the graph of $h$.

Proof. It is well-known that WF is $\Pi_{1}^{1}$-complete in $\mathscr{T}$ and that the height $T \mapsto \operatorname{ht}(T)$ is a $\Pi_{1}^{1}$ norm on WF. So if we define

$$
\begin{aligned}
& \Gamma_{0}:=\left\{(S, T) \in \mathscr{T} \times \mathscr{T}: S \in \mathrm{WF} \text { and } \operatorname{ht}(S)<^{*} \operatorname{ht}(T)\right\}, \\
& \Gamma_{1}:=\left\{(S, T) \in \mathscr{T} \times \mathscr{T}: T \in \mathrm{WF} \text { and } \operatorname{ht}(T) \leq^{*} \operatorname{ht}(S)\right\},
\end{aligned}
$$

we see as in Lemma 12 that the pair $\left(\Gamma_{0}, \Gamma_{1}\right)$ is complete in $\mathscr{T} \times \mathscr{T}$. So there exists a continuous mapping $\varphi: X \rightarrow \mathscr{T} \times \mathscr{T}$ which reduces $\left(C_{0}, C_{1}\right)$ to $\left(\Gamma_{0}, \Gamma_{1}\right)$. It is enough to prove the lemma for the pair $\left(\Gamma_{0}, \Gamma_{1}\right)$ since if $\psi: \Gamma_{0} \rightarrow \omega^{\omega}$ has the required property for the pair $\left(\Gamma_{0}, \Gamma_{1}\right)$, so does $h=\psi \circ \varphi$ for the pair $\left(C_{0}, C_{1}\right)$. Indeed assume that the sequence $\left(x_{j}\right)$ in $C_{0}$ converges to $x \in C_{1}$ and that $\left(h\left(x_{j}\right)\right)$ converges to $\alpha \in \omega^{\omega}$. Then $\left(\beta_{j}\right)=\left(\varphi\left(x_{j}\right)\right)$ is a sequence in $\Gamma_{0}$ which converges to $\beta=\varphi(x) \in \Gamma_{1}$ and $\psi\left(\beta_{j}\right) \rightarrow \alpha$. So $(\beta, \alpha)$ is a cluster point of the graph of $\psi$ with $\beta \in \Gamma_{1}$, in contradiction with the hypothesis on $\psi$.

Consider the following open game $G(S, T)$ with parameters $S$ and $T$ in $\mathscr{T}$ : Player I plays integers $m_{0}, m_{1}, \ldots$ and Player II plays integers $n_{0}, n_{1}, \ldots$ with the rule:

" $\left\langle n_{0}, n_{1}, \ldots, n_{k-1}\right\rangle$ must be in $S$ whenever $\left\langle m_{0}, m_{1}, \ldots, m_{k-1}\right\rangle$ is in $T$ ".

And Player II wins a run if it respects the rule forever.

We claim that Player I has a winning strategy in $G(S, T)$ if $(S, T) \in \Gamma_{0}$. As usual, for $T \in \mathscr{T}$ and $t \in \omega^{<\omega}$ we denote by $T_{t}$ the tree $\left\{s \in \omega^{<\omega}\right.$ : $\left.t^{\frown} s \in T\right\}$. Since

$$
\operatorname{ht}(T)=\operatorname{ht}\left(T_{\emptyset}\right)=\sup _{m}\left(\mathrm{ht}\left(T_{m}\right)+1\right)>\operatorname{ht}(S)
$$

there is an $m_{0}$ such that $\operatorname{ht}\left(T_{m_{0}}\right)+1>\operatorname{ht}(S)$, hence $\operatorname{ht}\left(T_{m_{0}}\right) \geq \operatorname{ht}(S)>$ $\operatorname{ht}\left(S_{n_{0}}\right)$ since $S$ is well-founded.

In the same way, if Player I has played $t=\left\langle m_{0}, m_{1}, \ldots, m_{k-1}\right\rangle$ and Player II has played $s=\left\langle n_{0}, n_{1}, \ldots, n_{k-1}\right\rangle$ in such a way that $\operatorname{ht}\left(T_{t}\right)>$ $\operatorname{ht}\left(S_{s}\right)$, we have again

$$
\operatorname{ht}\left(T_{t}\right)=\sup _{m}\left(\mathrm{ht}\left(T_{t-m}\right)+1\right)>\operatorname{ht}\left(S_{s}\right),
$$

hence Player I can find and play an integer $m_{k}$ such that $\left\langle m_{0}, m_{1}, \ldots, m_{k}\right\rangle \in T$ and $\operatorname{ht}\left(T_{t-m_{k}}\right) \geq \operatorname{ht}\left(S_{s}\right)>\operatorname{ht}\left(S_{s \frown n_{k}}\right)$. Since the sequence $\left(\operatorname{ht}\left(S_{\left\langle n_{0}, n_{1}, \ldots, n_{k}\right\rangle}\right)\right)$ 
of ordinals is decreasing, there must be a least integer $k$ with $\left\langle n_{0}, n_{1}, \ldots, n_{k}\right\rangle$ $\notin S$ whereas $\left\langle m_{0}, m_{1}, \ldots, m_{k}\right\rangle \in T$, and Player I wins.

As in Lemma 7 it follows from Martin's theorem that there is a bianalytic function $(S, T) \mapsto \sigma_{S, T}$ on $\Gamma_{0}$ which assigns to each $(S, T)$ a winning strategy for Player I in $G(S, T)$. Viewing a strategy $\sigma$ as a function $\left\langle n_{0}, n_{1}, \ldots, n_{k-1}\right\rangle \mapsto m_{k}$ from Seq $=\omega^{<\omega}$ to $\omega$, we identify the set $\Sigma$ of I-strategies with $\omega^{\text {Seq }} \simeq \omega^{\omega}$. We now have to prove that if $\left(S^{(j)}\right)$ and $\left(T^{(j)}\right)$ are sequences of trees converging to $S$ and $T$ respectively, if $\left(S^{(j)}, T^{(j)}\right) \in \Gamma_{0}$ and if $\sigma^{(j)}=\sigma_{S^{(j)}, T^{(j)}}$ converges to $\sigma \in \Sigma$, then $(S, T)$ cannot belong to $\Gamma_{1}$.

Assume towards a contradiction that $(S, T) \in \Gamma_{1}$. Then $T$ is well-founded and $\operatorname{ht}(T) \leq * \operatorname{ht}(S)$. We define sequences of integers $\left(m_{k}\right)$ and $\left(n_{k}\right)$ such that, for all $k$,

$$
m_{k}=\sigma\left(\left\langle n_{0}, n_{1}, \ldots, n_{k-1}\right\rangle\right) \quad \text { and } \operatorname{ht}\left(T_{\left\langle m_{0}, m_{1}, \ldots, m_{k}\right\rangle}\right)<\operatorname{ht}\left(S_{\left\langle n_{0}, n_{1}, \ldots, n_{k-1}\right\rangle}\right) .
$$

Indeed, if $\operatorname{ht}\left(T_{\left\langle m_{0}, m_{1}, \ldots, m_{k}\right\rangle}\right)<\operatorname{ht}\left(S_{\left\langle n_{0}, n_{1}, \ldots, n_{k-1}\right\rangle}\right)$, we have

$$
\operatorname{ht}\left(T_{\left\langle m_{0}, m_{1}, \ldots, m_{k}\right\rangle}\right)<\operatorname{ht}\left(S_{\left\langle n_{0}, n_{1}, \ldots, n_{k-1}\right\rangle}\right)=\sup _{n}\left(\operatorname{ht}\left(S_{\left\langle n_{0}, n_{1}, \ldots, n_{k-1}\right\rangle}{ }_{n}\right)+1\right)
$$

and there is an $n_{k}$ such that $\operatorname{ht}\left(T_{\left\langle m_{0}, m_{1}, \ldots, m_{k}\right\rangle}\right)<\operatorname{ht}\left(S_{\left\langle n_{0}, n_{1}, \ldots, n_{k}\right\rangle}\right)+1$. Then for $m_{k+1}=\sigma\left(\left\langle n_{0}, n_{1}, \ldots, n_{k}\right\rangle\right)$ we get

$$
\operatorname{ht}\left(T_{\left\langle m_{0}, m_{1}, \ldots, m_{k+1}\right\rangle}\right)<\operatorname{ht}\left(T_{\left\langle m_{0}, m_{1}, \ldots, m_{k}\right\rangle}\right) \leq \operatorname{ht}\left(S_{\left\langle n_{0}, n_{1}, \ldots, n_{k}\right\rangle}\right)
$$

and this allows us to pursue the inductive construction. And since $T$ is well-founded there is some $k$ such that $\left\langle m_{0}, m_{1}, \ldots, m_{k+1}\right\rangle \notin T$ whereas $\left\langle n_{0}, n_{1}, \ldots, n_{k}\right\rangle \in S$. Let $k$ be the least with this property. Since $\left(S^{(j)}\right)$ converges to $S,\left(T^{(j)}\right)$ converges to $T$ and $\left(\sigma^{(j)}\right)$ converges to $\sigma$, for all large enough $j$ we have $\left\langle n_{0}, n_{1}, \ldots, n_{p}\right\rangle \in S^{(j)}$ for $p \leq k,\left\langle m_{0}, m_{1}, \ldots, m_{p}\right\rangle \in T^{(j)}$ for $p \leq k,\left\langle m_{0}, m_{1}, \ldots, m_{k+1}\right\rangle \notin T^{(j)}$ and $m_{p}=\sigma^{(j)}\left(\left\langle n_{0}, n_{1}, \ldots, n_{p-1}\right\rangle\right)$ for $p \leq k+1$, in contradiction with the choice of $\sigma^{(j)}$ as a winning strategy in $G\left(S^{(j)}, T^{(j)}\right)$. This contradiction completes the proof of Lemma 13 .

Lemma 14. Let $X$ be a Polish space and $\left(C_{0}, C_{1}\right)$ a disjoint pair of coanalytic subsets of $X$. Then there exists a bianalytic function $h: C_{0} \rightarrow \omega^{\omega}$ such that no point of $C_{1} \times \omega^{\omega}$ lies in the closure of the graph of $h$.

Proof. Define $C=C_{0} \cup C_{1}$. Since $X$ is Polish there exists a closed subset $X^{*}$ of $\omega^{\omega}$ and a continuous bijection $\varphi$ from $X^{*}$ onto $X$. Then $\varphi^{-1}$ is Borel from $X$ to $X^{*}$, and so is $h_{0}=\varphi^{-1}{ }_{\mid C}: C \rightarrow X^{*} \subset \omega^{\omega}$. Define $C_{i}^{*}=\varphi^{-1}\left(C_{i}\right)$ for $i=0,1$. Then $\left(C_{0}^{*}, C_{1}^{*}\right)$ is a disjoint pair of coanalytic subsets of $X^{*}$. Applying Lemma 13 to $\left(C_{0}^{*}, C_{1}^{*}\right)$ we get a bianalytic function $h^{*}: C_{0}^{*} \rightarrow \omega^{\omega}$ such that the graph of $h^{*}$ has no cluster point in $C_{1}^{*} \times \omega^{\omega}$. Put $h_{1}=h^{*} \circ h_{0}$ : $C_{0} \rightarrow \omega^{\omega}$ and $h=h_{0} \times h_{1}: C \rightarrow \omega^{\omega} \times \omega^{\omega} \simeq \omega^{\omega}$.

If $\left(x_{j}\right)$ were a sequence in $C_{0}$ converging to $x \in C_{1}$ with $h\left(x_{j}\right) \rightarrow\left(x^{*}, \alpha\right)$ we would have $x_{j}^{*}:=h_{0}\left(x_{j}\right) \in C_{0}^{*}, x_{j}^{*} \rightarrow x^{*}$ and $h^{*}\left(x_{j}^{*}\right) \rightarrow \alpha$, hence $x_{j}=$ 
$\varphi\left(x_{j}^{*}\right) \rightarrow \varphi\left(x^{*}\right)$. This would show that $\varphi\left(x^{*}\right)=x \in C_{1}$ hence that $x^{*} \in C_{1}^{*}$, in contradiction with the choice of $h^{*}$.

Lemma 15. Let $X$ be a Polish space and $\left(C_{0}, C_{1}\right)$ be a disjoint pair of coanalytic subsets of $X$. Denote by $C$ the set $C_{0} \cup C_{1}$ and by $g: C \rightarrow\{0,1\}$ the bianalytic function defined by $g(x)=0 \Leftrightarrow x \in C_{0}$. Then there exists a bianalytic function $h: C \rightarrow \omega^{\omega}$ such that for every sequence $\left(x_{j}\right)$ in $C$ converging to some $x \in C$, if $\left(g\left(x_{j}\right)\right)$ converges to $a \in\{0,1\}$ and $\left(h\left(x_{j}\right)\right)$ converges to some $\alpha \in \omega^{\omega}$ then $g(x)=a$.

Proof. Applying Lemma 14 to $\left(C_{0}, C_{1}\right)$ and to $\left(C_{1}, C_{0}\right)$ we get bianalytic functions $h_{0}: C_{0} \rightarrow \omega^{\omega}$ and $h_{1}: C_{1} \rightarrow \omega^{\omega}$ such that the closure of the graph of $h_{i}$ is disjoint from $C_{1-i} \times \omega^{\omega}$ for $i=0,1$. Define then $h: C \rightarrow \omega^{\omega}$ by

$$
h(x)= \begin{cases}h_{0}(x) & \text { if } x \in C_{0}, \\ h_{1}(x) & \text { if } x \in C_{1} .\end{cases}
$$

Clearly, $h$ is $\boldsymbol{\Delta}_{1}^{1}$ on $C$. Moreover, if the sequence $\left(x_{j}\right)$ converges to $x \in C$ with $g\left(x_{j}\right) \rightarrow a \in\{0,1\}$ and $h\left(x_{j}\right) \rightarrow \alpha \in \omega^{\omega}$, for $j$ large enough we have $x_{j} \in C_{a}$ and $h\left(x_{j}\right)=h_{a}\left(x_{j}\right) \rightarrow \alpha$. We conclude that $x \notin C_{1-a}$, hence $x \in C_{a}$ and $g(x)=a$.

Lemma 16. Let $X$ be a Polish space and $\left(C_{0}, C_{1}\right)$ be a disjoint pair of coanalytic subsets of $X$. Denote by $C$ the set $C_{0} \cup C_{1}$ and by $g: C \rightarrow\{0,1\}$ the bianalytic function defined by $g(x)=0 \Leftrightarrow x \in C_{0}$. Then there exists a bianalytic function $h: C \rightarrow \omega^{\omega}$ such that the function $g \times h: C \rightarrow\{0,1\} \times \omega^{\omega}$ has a closed graph.

Proof. Let $\left(W_{k}\right)_{k \in \omega}$ be a sequence of clopen subsets of $\omega^{\omega}$ separating the points of $\omega^{\omega}$. We can define inductively for $s \in$ Seq bianalytic functions $g_{s}: C \rightarrow\{0,1\}$ and $h_{s}: C \rightarrow \omega^{\omega}$ in such a way that

(i) $g_{\emptyset}=g$,

(ii) $g_{s} \frown k(x)=1 \Leftrightarrow h_{s}(x) \in W_{k}$,

(iii) for every sequence $\left(x_{j}\right)$ converging to $x$ in $C$, if $\left(g_{s}\left(x_{j}\right)\right)$ converges to $a$ in $\{0,1\}$ and $\left(h_{s}\left(x_{j}\right)\right)$ converges to $\alpha$ in $\omega^{\omega}$ then $g_{s}(x)=a$.

Indeed, if $g_{s}$ is defined, we get $h_{s}$ by Lemma 15 . And if $h_{s}$ is $\boldsymbol{\Delta}_{1}^{1}$, so are the functions $g_{s \frown k}=\mathbf{1}_{W_{k}} \circ h_{s}$, where $\mathbf{1}_{W_{k}}$ is the characteristic function of $W_{k}$, which is continuous on $\omega^{\omega}$.

Then if we define $h=\prod_{s \in \text { Seq }} h_{s}: C \rightarrow\left(\omega^{\omega}\right)^{\text {Seq }} \simeq \omega^{\omega}$, the function $h$ is $\boldsymbol{\Delta}_{1}^{1}$. Moreover, if $\left(x_{j}\right)$ is a sequence in $C$ converging to $x \in C$ with

$$
g_{\emptyset}\left(x_{j}\right)=g\left(x_{j}\right) \rightarrow a_{\emptyset}:=a \in\{0,1\} \quad \text { and } \quad h\left(x_{j}\right) \rightarrow \beta=\left(\alpha_{s}\right)_{s \in \text { Seq }},
$$

we have $h_{s}\left(x_{j}\right) \rightarrow \alpha_{s}$ for all $s \in$ Seq, hence $g_{s \frown k}\left(x_{j}\right) \rightarrow a_{s \frown k}:=\mathbf{1}_{W_{k}}\left(\alpha_{s}\right)$ for all $s \in$ Seq and all $k$. 
For all $s$ we have $g_{s}\left(x_{j}\right) \rightarrow a_{s}$ and $h_{s}\left(x_{j}\right) \rightarrow \alpha_{s}$, hence $g_{s}(x)=a_{s}$. In particular, $g(x)=g_{\emptyset}(x)=a_{\emptyset}=a$. And if we had $h_{s}(x) \neq \alpha_{s}$ for some $s \in$ Seq there would exist some $k$ such that $\mathbf{1}_{W_{k}}\left(h_{s}(x)\right) \neq \mathbf{1}_{W_{k}}\left(\alpha_{s}\right)$, hence $g_{s \frown k}(x) \neq a_{s \frown k}$, a contradiction. We conclude that $g(x)=a$ and $h(x)=\beta$, hence the graph of $g \times h$ is closed.

Theorem 17. Let $X$ and $Y$ be Polish spaces, $C$ be a coanalytic subset of $X$ and $g: C \rightarrow Y$ be a $\boldsymbol{\Delta}_{1}^{1}$ function. Then there exists a $\boldsymbol{\Delta}_{1}^{1}$ function $h: C \rightarrow \omega^{\omega}$ such that the graph of $g \times h$ is closed in $C \times Y \times \omega^{\omega}$.

Proof. There exists a closed subset $Y^{*}$ of $\omega^{\omega}$, a continuous bijection $\varphi: Y^{*} \rightarrow Y$ and a homeomorphic embedding $\varrho$ of $Y^{*}$ into $2^{\omega}$. Then $\psi=$ $\varphi^{-1}: Y \rightarrow Y^{*}$ is Borel, $\varrho \circ \psi \circ g: C \rightarrow 2^{\omega}$ is bianalytic, and so are the coordinate functions $g_{n}: C \rightarrow\{0,1\}$ of $\varrho \circ \psi \circ g$. Applying Lemma 16 to each $g_{n}$ we find bianalytic functions $h_{n}: C \rightarrow \omega^{\omega}$ such that the graph of $g_{n} \times h_{n}$ is closed in $C \times\{0,1\} \times \omega^{\omega}$ for all $n$.

Define then $h=(\psi \circ g) \times \prod_{n} h_{n}: C \rightarrow \omega^{\omega} \times\left(\omega^{\omega}\right)^{\omega} \simeq \omega^{\omega}$. If the sequence $\left(x_{j}\right)$ converges in $C$ to $x$ with $y_{j}:=g\left(x_{j}\right) \rightarrow y$ and $h\left(x_{j}\right) \rightarrow$ $\beta=\left(y^{*},\left(\alpha_{n}\right)_{n \in \omega}\right)$, the sequence $\left(y_{j}^{*}\right):=\left(\psi \circ g\left(x_{j}\right)\right)$ converges to $y^{*}$. Thus $y_{j}=\varphi\left(y_{j}^{*}\right) \rightarrow \varphi\left(y^{*}\right)$, and this shows that $\varphi\left(y^{*}\right)=y$, hence $y^{*}=\psi(y)$. Moreover $\varrho \circ \psi \circ g\left(x_{j}\right)=\varrho\left(y_{j}^{*}\right) \rightarrow \varrho\left(y^{*}\right)=\left(a_{n}\right) \in 2^{\omega}$ and $\varrho\left(y^{*}\right)=\varrho \circ \psi(y)$. Hence $g_{n}\left(x_{j}\right) \rightarrow a_{n}$ and $h_{n}\left(x_{j}\right) \rightarrow \alpha_{n}$. Since the graph of $g_{n} \times h_{n}$ is closed we get $a_{n}=g_{n}(x)$ and $h_{n}(x)=\alpha_{n}$ for all $n$, hence $\varrho \circ \psi \circ g(x)=\varrho \circ \psi(y)$ and $h(x)=\beta$. Thus $g(x)=y$ and $h(x)=\beta$, and this shows that $g \times h$ has a closed graph.

Corollary 18. Let $X$ be a separable metrizable space, $Y$ be a Polish space and $g$ be a mapping from $X$ to $Y$. Then $g$ is bianalytic if and only if there exists a Polish space $Z$ and a mapping $h: X \rightarrow Z$ such that $g \times h$ : $X \rightarrow Y \times Z$ has a closed graph in $X \times Y \times Z$.

Proof. Assume first that there exists $h: X \rightarrow Z$ such that $g \times h$ has a closed graph $G$. Let $\widehat{X}$ be the completion of $X$ and $\bar{G}$ be the closure of $G$ in $\widehat{X} \times(Y \times Z)$. Since $G$ is closed in $X \times(Y \times Z)$ we have $X \subset U(\bar{G})$ and the mapping $x \mapsto(g(x), h(x))$ is bianalytic on $U(\bar{G})$ by Theorem 3 . It follows that $g$ is $\boldsymbol{\Delta}_{1}^{1}$ on $X$.

Conversely, if $g$ is $\boldsymbol{\Delta}_{1}^{1}$ on $X$, it has a $\boldsymbol{\Delta}_{1}^{1}$ extension $\widetilde{g}$ onto a coanalytic subset $C$ of $\widehat{X}$ containing $X$. Applying Theorem 17 we get a $\Delta_{1}^{1}$ mapping $h: C \rightarrow \omega^{\omega}$ such that $\widetilde{g} \times h$ has a closed graph on $C$. It follows that the graph of $g \times h_{\mid X}$ is closed in $X \times Y \times \omega^{\omega}$.

\section{Some examples of complete pairs of coanalytic sets}

Lemma 19. Let $X$ be a Polish space and $\left(C_{0}, C_{1}\right)$ be a disjoint pair of coanalytic subsets of $X$. Denote by $\pi: X \times 2^{\omega} \rightarrow X$ the projection $(x, \alpha) \mapsto x$. 
Then there exists a $\Pi_{2}^{0}$ subset $B$ of $X \times 2^{\omega}$ such that $C_{0} \subset X \backslash \pi(B)$ and $C_{1} \subset U(B)$.

Proof. Let $C=C_{0} \cup C_{1}$ and $g: C \rightarrow\{0,1\}$ be the bianalytic function defined by $g(x)=0 \Leftrightarrow x \in C_{0}$. By Lemma 16 there exists a $\boldsymbol{\Delta}_{1}^{1}$ function $h: C \rightarrow \omega^{\omega}$ such that the graph $G$ of $g \times h$ is closed in $C \times\{0,1\} \times \omega^{\omega}$. Then for $x \in C, a \in\{0,1\}$ and $\alpha \in \omega^{\omega}$ we have

$$
(x, a, \alpha) \in \bar{G} \Leftrightarrow a=g(x) \text { and } \alpha=h(x) .
$$

Denote by $\varphi$ a homeomorphism from $\omega^{\omega}$ to a $\Pi_{2}^{0}$ subset $Y$ of $2^{\omega}$ (e.g. $2^{\omega} \backslash \mathbb{Q}$ ) and define

$$
B=\left\{(x, \beta) \in X \times Y:\left(x, 1, \varphi^{-1}(\beta)\right) \in \bar{G}\right\} .
$$

Clearly $B$ is closed in $X \times Y$ hence $\Pi_{2}^{0}$ in $X \times 2^{\omega}$. Moreover, for $x \in C_{0}$ there is exactly one point $(x, a, \alpha) \in \bar{G}$ and $a=0$. Thus $x \notin \pi(B)$. And for $x \in C_{1}$ there is exactly one point $(x, a, \alpha) \in \bar{G}$, and $a=1$. So $x \in U(B)$.

Theorem 20. Let $X$ be a zero-dimensional Polish space and $\left(C_{0}, C_{1}\right)$ a disjoint pair of coanalytic subsets of $X$. Then there exists a continuous function $\Phi: X \rightarrow \mathscr{K}\left(2^{\omega}\right)$ such that $\Phi(x) \in \mathscr{K}(\mathbb{Q})$ if $x \in C_{0}, \Phi(x) \in \mathscr{K}^{*}(\mathbb{Q})$ if $x \in C_{1}$ and $\Phi(x) \notin \mathscr{K}_{\omega}\left(2^{\omega}\right)$ if $x \notin C_{0} \cup C_{1}$.

Proof. By Lemma 19 there exists a $\Pi_{2}^{0}$ subset $B$ of $X \times 2^{\omega}$ such that $C_{0} \cap \pi(B)=\emptyset$ and $C_{1} \subset U(B)$. Since $X \times 2^{\omega}$ is zero-dimensional there is a continuous function $f: X \times 2^{\omega} \rightarrow 2^{\omega}$ reducing the $\Pi_{2}^{0}$ set $B$ to $2^{\omega} \backslash \mathbb{Q}$. Then for $x \in X$ we put

$$
\Phi^{\prime}(x)=f\left(\{x\} \times 2^{\omega}\right) \in \mathscr{K}\left(2^{\omega}\right) .
$$

Since the mapping $x \mapsto\{x\} \times 2^{\omega}$ is continuous from $X$ to $\mathscr{K}\left(X \times 2^{\omega}\right)$, it follows that $\Phi^{\prime}$ is continuous.

If $x \in C_{0}$ then $x \notin \pi(B)$, hence $\{x\} \times 2^{\omega} \subset f^{-1}(\mathbb{Q})$ and $\Phi^{\prime}(x) \in \mathscr{K}(\mathbb{Q})$. If $x \in C_{1}$ then $x \in U(B)$, hence $\left(\{x\} \times 2^{\omega}\right) \backslash f^{-1}(\mathbb{Q})$ has exactly one point; it follows that $\Phi^{\prime}(x) \in \mathscr{K}^{*}(\mathbb{Q})$.

Moreover, $C=C_{0} \cup C_{1}$ is coanalytic, and there exists a continuous mapping $\Phi^{\prime \prime}$ from $X$ to $\mathscr{K}\left(2^{\omega}\right)$ such that $\Phi^{\prime \prime}(x) \in \mathscr{K}(\mathbb{Q})$ for all $x$ in $C$ and $\Phi^{\prime \prime}(x) \notin \mathscr{K}_{\omega}\left(2^{\omega}\right)$ for all $x$ outside $C$ (see for example Lemma 5.1 .7 in [1]).

Then the mapping $\Phi: x \mapsto \Phi^{\prime}(x) \cup \Phi^{\prime \prime}(x)$ is clearly continuous from $X$ to $\mathscr{K}\left(2^{\omega}\right)$ and has the required properties.

We immediately deduce from this theorem the next two corollaries.

COROLlaRY 21. The pair $\left(\mathscr{K}(\mathbb{Q}), \mathscr{K}^{*}(\mathbb{Q})\right)$ of coanalytic subsets of $\mathscr{K}\left(2^{\omega}\right)$ is complete.

Corollary 22. The pair $\left(\mathscr{K}(\mathbb{Q}), \mathscr{K}_{\omega}\left(2^{\omega}\right) \backslash \mathscr{K}(\mathbb{Q})\right)$ of coanalytic subsets of $\mathscr{K}\left(2^{\omega}\right)$ is complete. 
We give one more example of a complete disjoint pair of coanalytic subsets.

Theorem 23. The pair (WF, $\mathrm{WF}^{*}$ ) of coanalytic subsets of $\mathscr{T}$ is complete.

Proof. Let $X$ be a closed subset of $\omega^{\omega}$ and $\left(C_{0}, C_{1}\right)$ a disjoint pair of coanalytic subsets of $X$. Let $C=C_{0} \cup C_{1}$ and $g: C \rightarrow\{0,1\}$ be the bianalytic function defined by $g(x)=0 \Leftrightarrow x \in C_{0}$. By Lemma 16 there exists a $\Delta_{1}^{1}$ function $h: C \rightarrow \omega^{\omega}$ such that the graph $G$ of $g \times h$ is closed in $C \times\{0,1\} \times \omega^{\omega}$. Then for $x \in C, a \in\{0,1\}$ and $\alpha \in \omega^{\omega}$ we have

$$
(x, a, \alpha) \in \bar{G} \Leftrightarrow a=g(x) \text { and } \alpha=h(x) .
$$

Then define $F^{\prime}:=\left\{(x, \alpha) \in X \times \omega^{\omega}:(x, 1, \alpha) \in \bar{G}\right\}$. Clearly, $F^{\prime}$ is closed in $X \times \omega^{\omega}$ and we have $C_{0} \cap \pi\left(F^{\prime}\right)=\emptyset$ and $C_{1} \subset U\left(F^{\prime}\right)$. Since $C$ is coanalytic there exists a closed subset $H$ of $X \times \omega^{\omega}$ such that $\pi(H)=X \backslash C$. Then $H \times \omega^{\omega}$ is a closed subset of $\left(X \times \omega^{\omega}\right) \times \omega^{\omega} \simeq X \times\left(\omega^{\omega} \times \omega^{\omega}\right) \simeq X \times \omega^{\omega}$. Thus there exists a closed subset $F^{\prime \prime}$ of $X \times \omega^{\omega}$ such that $\pi\left(F^{\prime \prime}\right)=X \backslash C$ and the fiber $F^{\prime \prime}(x)$ is uncountable for $x \notin C$.

The closed subset $F=F^{\prime} \cup F^{\prime \prime}$ of $X \times \omega^{\omega}$ then satisfies $F(x)=\emptyset$ if $x \in C_{0}, F(x)$ is a singleton if $x \in C_{1}$, and $F(x)$ is uncountable if $x \in X \backslash C$. Let $T$ be a tree on $\omega \times \omega$ such that $\lceil T\rceil=F \subset X \times \omega^{\omega} \subset \omega^{\omega} \times \omega^{\omega}$. Define a continuous mapping from $X$ to $\mathscr{T}$ by

$$
T(x)=\{s \in \text { Seq }: \exists t \prec x|t|=|s| \text { and }(t, s) \in T\} .
$$

It is easily checked that the set $\lceil T(x)\rceil$ of infinite branches of the tree $T(x)$ is equal to the fiber $F(x)$. So $T(x) \in \mathrm{WF}$ if $x \in C_{0}, T(x) \in \mathrm{WF}^{*}$ if $x \in C_{1}$ and $T(x) \notin \mathrm{WF} \cup \mathrm{WF}^{*}$ if $x \notin C$. And this shows that the mapping $x \mapsto T(x)$ is a continuous reduction of the pair $\left(C_{0}, C_{1}\right)$ to the pair $\left(\mathrm{WF}, \mathrm{WF}^{*}\right)$. It follows that the pair $\left(\mathrm{WF}, \mathrm{WF}^{*}\right)$ is complete.

6. Complete sequences of coanalytic sets. More generally, one can consider disjoint sequences of coanalytic sets instead disjoint pairs. We will say that the sequence $\left(C_{n}\right)$ of pairwise disjoint coanalytic subsets of the Polish space $Y$ is complete if for every Polish zero-dimensional space $X$ and every sequence $\left(D_{n}\right)$ of pairwise disjoint coanalytic subsets of $X$ there exists a continuous function $f: X \rightarrow Y$ such that $D_{n}=f^{-1}\left(C_{n}\right)$ for all $n$.

For every $n \in \omega$ we denote by $\mathrm{WF}^{(n)}$ the set of trees on $\omega$ having exactly $n$ infinite branches. So $\mathrm{WF}^{(0)}=\mathrm{WF}$ and $\mathrm{WF}^{(1)}=\mathrm{WF}^{*}$. We intend to prove that the sequence $\left(\mathrm{WF}^{(n)}\right)_{n \in \omega}$ is complete.

Lemma 24. Let $Y$ and $Z$ be Polish spaces, and $X$ be a Borel subset of $Y \times Z$. Then, for every integer $n$, the set $U_{n}(X)$ of points $y \in Y$ such that the fiber $X(y)$ has exactly $n$ points in $Z$ is coanalytic. 
Proof. This is already proved for $n=0$ and $n=1$. Let $\left(W_{k}\right)$ be a countable basis of the topology of $Z$, and

$$
D_{n}=\left\{\left(k_{0}, k_{1}, \ldots, k_{n-1}\right) \in \omega^{n}: W_{k_{i}} \cap W_{k_{j}}=\emptyset \text { for } 0 \leq i<j<n\right\} .
$$

Let $\pi: Y \times Z \rightarrow Y$ denote the first projection. Define for all $k$ the coanalytic set $E_{k}$ by $E_{k}:=U\left(X \cap\left(Y \times W_{k}\right)\right)$ and for $\left(k_{0}, k_{1}, \ldots, k_{n-1}\right) \in D_{n}$ the analytic set $A_{\left(k_{0}, k_{1}, \ldots, k_{n-1}\right)}$ by

$$
A_{\left(k_{0}, k_{1}, \ldots k_{n-1}\right)}=\pi\left(X \cap\left(Y \times\left(Z \backslash \bigcup_{j<n} W_{k_{j}}\right)\right)\right) .
$$

It is clear that a point $y$ lies in $U_{n}(X)$ if and only if there are $n$ pairwise disjoint open sets $\left(V_{0}, V_{1}, \ldots, V_{n-1}\right)$ such that $X(y)$ contains exactly one point in each $V_{j}$ and no point outside $\bigcup_{j<n} V_{j}$. So

$$
U_{n}(X)=\bigcup_{\left(k_{0}, k_{1}, \ldots, k_{n-1}\right) \in D_{n}}\left(\bigcap_{j<n} E_{k_{j}} \backslash A_{\left(k_{0}, k_{1}, \ldots, k_{n-1}\right)}\right),
$$

and this shows that $U_{n}(X) \in \Pi_{1}^{1}$.

LEMMA 25. For all integer $n, \mathrm{WF}^{(n)}$ is a coanalytic subset of $\mathscr{T}$.

Proof. Let $X$ be the set $\left\{(T, \alpha) \in \mathscr{T} \times \omega^{\omega}: \alpha \in\lceil T\rceil\right\}$. Then $X$ is closed in $\mathscr{T} \times \omega^{\omega}$ and $\mathrm{WF}^{(n)}=U_{n}(X)$ for all $n \in \omega$. So the conclusion follows immediately from Lemma 24 .

Lemma 26. Let $X$ be a Polish space and $\left(C_{n}\right)$ be a sequence of pairwise disjoint coanalytic subsets of $X$. Then there exists a closed subset $F$ of $X \times \omega^{\omega}$ such that $C_{n}=U_{n}(X)$ for all integer $n$.

Proof. Consider the bianalytic function $g$ defined on the coanalytic set $C=\bigcup_{n} C_{n}$ by $g(x)=n \Leftrightarrow x \in C_{n}$. By Theorem 17 there is a bianalytic function $h: C \rightarrow \omega^{\omega}$ such that the graph $G$ of $g \times h$ is closed in $C \times \omega \times \omega^{\omega}$. Then $\bar{G}$ is closed in $X \times \omega \times \omega^{\omega}$ and we have $\bar{G}(x)=\{(n, h(x))\}$ for every $x \in C_{n}$. As in the proof of Theorem 23 we can find a closed subset $F^{\prime}$ of $X \times\left(\omega \times \omega^{\omega}\right)$ such that $F^{\prime}(x)=\emptyset$ for $x \in C$ and $F^{\prime}(x)$ is uncountable for $x \notin C$.

Then we define $F \subset X \times\left(\omega \times \omega^{\omega}\right) \simeq X \times \omega^{\omega}$ by

$$
(x, m, \alpha) \in F \Leftrightarrow\left((x, m, \alpha) \in F^{\prime}\right) \text { or }(\exists n n \leq m<2 n \text { and }(x, n, \alpha) \in \bar{G}) \text {. }
$$

It is easily checked that $F$ is closed, that $F(x) \supset F^{\prime}(x)$ is uncountable for $x \notin C$ and that, for $x \in C_{n}, F(x)=\{(m, h(x)): n \leq m<2 n\}$, hence has exactly $n$ points. Thus $U_{n}(X)=C_{n}$.

THEOREM 27. The sequence $\left(\mathrm{WF}^{(n)}\right)_{n \in \omega}$ of pairwise disjoint coanalytic subsets of $\mathscr{T}$ is complete. 
Proof. Let $X$ be a closed subset of $\omega^{\omega}$ and $\left(C_{n}\right)$ a sequence of pairwise disjoint coanalytic subsets of $X$. By Lemma 26 there is a closed subset $F$ of $X \times \omega^{\omega}$, hence of $\omega^{\omega} \times \omega^{\omega}$, such that $U_{n}(F)=C_{n}$ for all $n$. Let $T$ be a tree on $\omega \times \omega$ such that $F=\lceil T\rceil$. Then we define a continuous mapping from $X$ to $\mathscr{T}$ by

$$
T(x)=\{s \in \text { Seq }: \exists t \prec x \quad(t, s) \in T \text { and }|s|=|t|\} .
$$

Since $\lceil T(x)\rceil=F(x)$ for all $x \in X$ we have $x \in C_{n} \Leftrightarrow T(x) \in \mathrm{WF}^{(n)}$ for all $n$. Thus the mapping $x \mapsto T(x)$ is a continuous reduction of the sequence $\left(C_{n}\right)$ to the sequence $\left(\mathrm{WF}^{(n)}\right)$.

Since $X$ and the sequence $\left(C_{n}\right)$ are arbitrary this shows that the sequence $\left(\mathrm{WF}^{(n)}\right)$ is complete.

\section{References}

[1] G. Debs and J. Saint Raymond, Borel liftings of Borel sets: some decidable and undecidable statements, Mem. Amer. Math. Soc., to appear; preprint available at www.institut.math.jussieu.fr/ ${ }^{\sim}$ raymond/preprints/treerep.pdf.

[2] C. Dellacherie, Ensembles analytiques: théorèmes de séparation et applications, in: Séminaire de Probabilités IX, Lecture Notes in Math. 465, Springer, Berlin, 1975, 336-372.

[3] L. Harrington, Analytic determinacy and $0^{\sharp}$, J. Symbolic Logic 43 (1978), 685-693.

[4] A. Kechris, Classical Descriptive Set Theory, Springer, New York, 1995.

[5] Y. N. Moschovakis, Descriptive Set Theory, North-Holland, Amsterdam, 1980.

Analyse Fonctionnelle

Institut de Mathématique de Jussieu

Boîte 186

4 , place Jussieu

75252 Paris Cedex 05, France

E-mail: jsr@ccr.jussieu.fr

Received 19 September 2006;

in revised form 30 January 2007 